\title{
Connectivity analysis is essential to understand neurological disorders
}

\author{
James B. Rowe $e^{1,2,3 *}$ \\ Department of Clinical Neurosciences, University of Cambridge, Cambridge, UK \\ 2 Cognition and Brain Sciences Unit, Medical Research Council, Cambridge, UK \\ ${ }^{3}$ Behavioural and Clinical Neurosciences Institute, University of Cambridge, Cambridge, UK
}

\section{Edited by:}

Barry Horwitz, National Institutes of Health, USA

\section{Reviewed by:}

Barry Horwitz, National Institutes of Health, USA

Arun Bokde, Trinity College Dublin, Ireland

\section{*Correspondence:}

James B. Rowe, Cognition and Brain Sciences Unit, Medical Research Council, 15 Chaucer Road, Cambridge CB2 TEF, UK.

e-mail:james.rowe@mrc-cbu.cam. ac.uk
Neurological and neuropsychiatric disorders are major causes of morbidity worldwide. A systems level analysis including functional and structural neuroimaging is particularly useful when the pathology leads to disorders of higher order cognitive functions in human patients. However, an analysis that is restricted to regional effects is impoverished and insensitive, compared to the analysis of distributed brain networks. We discuss the issues to consider when choosing an appropriate connectivity method, and compare the results from several different methods that are relevant to $\mathrm{fMRI}$ and PET data. These include psychophysiological interactions in general linear models, structural equation modeling, dynamic causal modeling, and independent components analysis. The advantages of connectivity analysis are illustrated with a range of structural and neurodegenerative brain disorders. We illustrate the sensitivity of these methods to the presence or severity of disease and/or treatment, even where analyses of voxel-wise activations are insensitive. However, functional and structural connectivity methods should be seen as complementary to, not a substitute for, other imaging and behavioral approaches. The functional relevance of changes in connectivity, to motor or cognitive performance, are considered alongside the complex relationship between structural and functional changes and neuropathology. Finally some of the problems associated with connectivity analysis are discussed. We suggest that the analysis of brain connectivity is an essential complement to the analysis of regionally specific dysfunction, in order to understand neurological and neuropsychiatric disease, and to evaluate the mechanisms of effective therapies.

Keywords: effective connectivity, functional connectivity, structural equation modeling, dynamic causal modeling psychophysiological interactions, MRI

\section{INTRODUCTION}

Neurological and neuropsychiatric disorders are major causes of morbidity and mortality worldwide. They include developmental and degenerative processes, as well as focal brain injury from stroke or trauma, and are subject to many genetic and environmental influences. The heterogeneity and complexity of individual clinical syndromes reflect interactions among patterns of neuropathology, individual differences in premorbid function and the distributed functional anatomy of normal cognitive and motor processes. This presents a double challenge for clinical translational cognitive neuroscience - to define simultaneously the processes or systems underlying neuropsychiatric syndromes, and to understand their functional anatomical abnormalities.

For many years, a neuropsychological approach was the only way to dissociate functional components of behavioral syndromes, and often localize clinical phenomena to specific brain regions. With the advent of structural brain imaging, combined with behavioral analysis and larger case series, this approach remains informative (Mort et al., 2003; Sapir et al., 2007; Verdon et al., 2010). However, methods that identify localized or functionally segregated disease effects are fundamentally limited, for several reasons.

First and foremost is that cognitive processes depend fundamentally on interactions among multiple brain regions, rather than on isolated processes within regions. Even seemingly basic early sensory cortical functions are not simply determined by inputs from the periphery, but are modulated by back-projections within networks of primary, secondary, and association cortices. Neurological disorders that are manifest by dysfunction of distributed cognitive processes may arise from changes in the interactions (connectivity) within the cortical and subcortical networks, as well as isolated abnormalities within any one "node" of the network. This principle was established in early modern neurology by Lichtheim's anticipation and identification of conduction aphasia, and it remains highly relevant today.

Second, many neuropathologies are themselves distributed widely (Braak and Braak, 1997; Braak et al., 2006). The reasons for selective vulnerability of a subset of brain networks to disease are rarely clear cut, but a consequence of distributed pathology is that there may be severely impaired function of the network without consistent and sufficiently severe localized abnormalities to detect group-based localized deficits. This applies not only to neurodegenerative disorders such as Parkinson's disease (PD) but also multifocal cerebral insults such as subcortical ischemia or demyelination.

Third, a localized lesion may not cause relevant functional abnormalities in only its own location and immediate connections. There can also be remote consequences within the neural network in which the lesion is embedded. In complex networks, 
with reciprocal connections or large scale circuit loops, the local and remote changes in connectivity can be difficult to predict, in clinical (Sharma et al., 2009) and simulated data (Kim and Horwitz, 2009).

Several methods to study connectivity are commonly used at present (see Table 1). We begin by considering the choice of methods. Subsequent sections will emphasize the commonalities of methods, and the importance of using these methods in order to fully understand disorders of the nervous system, as well as effective treatments. The challenges of these complex network interactions apply not only to disease, but also the neural basis of healthy individual differences. Although this review focuses on neurological disorders, the principles are also relevant to individual differences in the healthy population.

\section{WHICH METHOD OF CONNECTIVITY SHOULD ONE CHOOSE?}

There are many available methods to study large scale neural networks from functional neuroimaging data (Table 1). I will illustrate several of them in this review including psychophysiological interactions (PPIs), structural equation modeling (SEM), dynamic causal modeling (DCM), independent components analysis (ICA), and partial least squares (PLS), with an emphasis on fMRI data in neurological conditions.

A distinction is often drawn between functional connectivity and effective connectivity. Functional connectivity refers to covariance over time among spatially distributed brain regions which may arise because they are part of a common network. However, it may also be observed in the absence of causal influences among the regions if there are common inputs from other areas; multifocal effects of drugs; or common sources of noise. In contrast, effective connectivity refers explicitly to the causal influences of one region over another (see Limitations, Counter Intuitive Results and Naive Expectations). It often also implies that these influences are related to the study paradigm or an intervention such as transcranial magnetic stimulation. Some methods are clearly designed to study effective connectivity, based on temporal precedence (e.g., GCM) or dynamic causal models (e.g., DCM). Other methods (e.g., PPIs)

\section{Table 1 | Glossary and outline of methods discussed.}

DCM Dynamic causal
modeling

GCM Granger causality modeling and Granger causality mapping

ICA Independent component analysis

PLS Partial least squares

PPI Psychophysiological interactions

RSN Resting state networks

SEM Structural equation modeling
A deterministic approach within a generative model that characterizes neural activity in terms of driving inputs to a distributed neural network, intrinsic connections, and linear or non-linear modulations of connectivity arising from tasks or neural activity (Friston et al., 2003). Critical features of DCM as implemented by SPM software are the simultaneous estimation of a forward model of neurovascular coupling and the interactions among network regions at the level of neuronal activity. These are estimated to optimize a free energy estimate of the log-evidence of a model in a Bayesian framework. DCM is currently applicable to single subject and group studies of $\mathrm{fMRI}$ and M/EEG data, with extensions able to incorporate multiple state representations at each region and stochastic or spontaneous activations. See www.fil.ion.ucl.ac.uk/spm

These methods examine connectivity in terms of "Granger causality" (Roebroeck et al., 2005, 2009a), emphasizing the role of temporal precedence in the inference of causality. They are closely related to multivariate autoregressive modeling, which like SEM has its roots in econometrics, and can be applied to test anatomically defined neural network models (Granger causality modeling), or explore the interactions between a source region all other regions (Granger causality mapping). An invaluable discussion of issues related to GCM for fMRI data is contained in the exchange between Friston and Roebroeck (see Friston, 2009; Roebroeck et al., 2009a,b). See www.brainvoyager.com Model-free $\mathrm{fMRl}$ analysis which may in some packages also estimate the number of interesting noise and signal sources in the data (McKeown et al., 1998; Beckmann et al., 2005). This approach does not assume anatomical connectivity or directionality of influences within the networks, but component networks can be mapped to task events or contexts. See www.fmrib.ox.ac.uk/fsl/melodic or afni.nimh.nih.gov/sscc/gangc/ica

Related to principal components analysis, PLS identifies functionally connected brain networks and can identify subject- or experimental-variables associated with them (Mclntosh et al., 1996; Mclntosh and Lobaugh, 2004) as well as identifying psychophysiological interactions. See www.rotman-baycrest.on.ca/

A general conceptual framework in which physiological interactions between regions are modulated by psychological or physiological contexts. It can be used to test hypotheses of effective connectivity (Friston et al., 1997), or explore functional connectivity. However, the term PPI is also used to refer to a specific implementation within general linear models (PPI-GLMs). These PPI-GLMs use moderator variables that express the interactions between regional activations and contexts (and higher order interactions with between-subjects factors like age or disease risk factors) (Buchel and Friston, 1997; Rowe et al., 2006; Passamonti et al., 2009). See www.fil.ion.ucl.ac.uk/spm

ICA of $\mathrm{FMRI}$ data acquired at rest identifies a small number $(10)$ of consistent spatially distributed covarying brain networks. One of these is also commonly identified by the brain state when not engaged in typical experimental tasks, known as the default mode network (DMN)

Introduced into neuroimaging from econometrics and social sciences for the analysis of brain effective connectivity analysis (McIntosh and Gonzalez-Lima, 1994; Mclntosh et al., 1994), to determine task-dependent (Mclntosh et al., 1994; Buchel and Friston, 1997; Honey et al., 2002) or group-dependent (Grafton et al., 1994; Horwitz et al., 1995; Rowe et al., 2002b) changes in a hypothesized causal structure formalized in a path model. Commonly implemented for $\mathrm{AMRI}$ data by LISREL or SPM toolbox software. 
can be used either to test causal models (effective connectivity) or to explore changes in task related covariance (functional connectivity). If functional connectivity is sufficient to generate or test hypotheses, then validated options include partial least squares (PLS) (McIntosh et al., 2004; McIntosh and Lobaugh, 2004), principal components analysis (PCA), or independent components analysis (ICA) (McKeown et al., 1998).

It is therefore necessary for each study to be clear about the hypotheses being tested and the biophysical interpretation of connectivity parameters (Buchel and Friston, 2000; Penny et al., 2004b; Ramnani et al., 2004; Lee et al., 2006; Friston, 2009; Roebroeck et al., 2009a,b; Cole et al., 2010). With this in mind, I will show these different methods can each contribute to our understanding of the complex network interactions in the brain; their relevance to neurological disease; and discuss how to choose an appropriate method.

Few studies directly compare multiple methods on the same data, despite the lack of consensus for many years (Horwitz, 2003). There are many reasons why two methods might differ in the inferred changes in connectivity, including a different biophysical interpretation of connectivity parameters and differential sensitivity to artifacts. Nonetheless, direct comparisons are interesting. Passamonti et al. (2008) directly compared psychophysiological interactions in general linear models (PPI-GLM) and dynamic causal modeling (DCM). They examined the connectivity between amygdala and medial frontal cortex, in relation to individual personality differences. The conclusions of these two methods were congruent. In the context of Parkinson's disease (PD), Palmer et al. (2009) compared SEM and multivariate autoregressive modeling. Both methods revealed similar dopamine-dependent group differences in connectivity, despite significant differences in methods and their interpretation. Using simulated data, Witt and Meyerand (2009) compared SEM, autoregression analysis, Granger causality modeling (GCM), and DCM and again found that each method was able to detect the underlying system dynamics (although GCM was adversely sensitive to differences in imaging and modeling parameters). Thus, methods do appear to yield consistent, although not identical results. However, different methods permit different inferences, and they are not all appropriate for every circumstance.

How then should one choose the right method to study the effects of disease or treatments? This depends on a number of practical considerations. First, what types of inference are relevant? For example, are inferences of directional and causal influences required? If so, then dynamic causal modeling (DCM) (Friston et al., 2003; Penny et al., 2004a; Sonty et al., 2007; Stephan et al., 2008; Rowe et al., 2010), SEM (McIntosh and Gonzalez-Lima, 1994; Buchel and Friston, 1997; Rowe et al., 2002b; Sharma et al., 2009) or Granger causality modeling (GCM) (Roebroeck et al., 2005) should be considered. Note that GCM of fMRI data is the youngest of these methods, and important theoretical considerations have been discussed recently (Friston, 2009; Roebroeck et al., 2009a,b). Alternatively, is it sufficient to establish differences in spatiotemporal covariance? If so, functional connectivity methods such as simple correlations analysis, partial least squares (PLS) (McIntosh et al., 2004; McIntosh and Lobaugh, 2004), principal components analysis or independent components analysis (McKeown et al., 1998) may be preferable to infer the presence or connectivity of spatiotemporally covarying networks. If one is able to predefine the specific experimental manipulations or disease factors that determine the changes in connectivity, these factors may be incorporated as hypothesized modulatory influences, e.g., in DCM, SEM or PPIGLMs. Alternatively, data driven approaches such as PLS or post hoc interrogation of independent components analysis may be used to identify relevant factors that define a network's function.

Second, the appropriate methods may be restricted by the clinical conditions, by disease-related confounding factors such as medication, or by the cognitive systems of interest. Some tasks are not well suited to temporally precise network models, e.g., tasks with long epochs without discrete events are not easily modeled accurately in DCM. Such tasks include imagination of movements without precise timing of imagined "events"; observation of video clips with imprecise timing of critical perceptions; or long working memory delays. Another problem arises from the use of a canonical hemodynamic response function to estimate interactions between task, context and disease, e.g., SEM or PPI-GLMs. These might be confounded by a disease or medication that fundamentally alters the hemodynamic response function (Iannetti and Wise, 2007). In contrast, other methods such as DCM, which estimate a subject-, session-, and region-specific forward model of the neurovascular BOLD response, can accommodate disease or drug dependent differences in hemodynamic response function. In addition, general linear models of psychophysiological interactions are less suited to rapid event-related designs, which might be required to study the psychological or behavioral phenomenon of relevance to the disease such as the response to unexpected events.

A third factor is the information already available from the literature. Can one justify a set of cognitive and anatomical models, within which to evaluate the effects of disease on network connectivity? If so, then models embodying anatomically defined regions of interest and the causal relations among them can be tested, e.g., DCM or SEM. In addition, DCM, can be used to compare the effects of disease or treatment within multiple plausible models, and identify the most likely causal network model for a given cognitive process even in heterogeneous groups with between-subject differences in network dynamics (Penny et al., 2004a; Stephan et al., 2009a). Alternatively, an exploratory approach may be required, using PPIs, GCM, or ICA of whole brain data. These methods can be applied without restricting the analysis of disease effects on connectivity to a predefined anatomical network and without defining unidirectional or bidirectional interactions among regions.

A related question is whether it is justified to restrict analysis to connectivity changes induced by experimental manipulations? Deterministic models, such as those embodied by DCM, are powerful tools to define linear or non-linear networks related to experimental tasks, and their interactions with psychological contexts, diseases or pharmacology. However, current versions of DCM do not support stochastic or spontaneous processes within defined networks. The latter call for SEM which includes spontaneous activity ("innovations") arising within the network, rather than external or driving inputs to the network. However, a variational Bayes approach to DCM can approximate non-linear and stochastic dynamic models (Stephan et al., 2008; Daunizeau et al., 2009), encompassing endogenous sources of changing connectivity. Alternatively, connectivity may be inferred from model independent 
functional connectivity methods such as independent components analysis (ICA) of "spontaneous" resting state fluctuations (see Distributed (dis-)Connectivity for Distributed Neuropathology). In brief, different methods are currently required to examine connectivity changes resulting from spontaneous processes compared to changes resulting from to experimental manipulations.

Whilst there are important theoretical considerations behind the right choice of method, each of the methods has been helpful in understanding the effects of disease. These methods may broadly agree, where commonalities are sufficient to enable direct comparisons. Often, the connectivity analysis is presented alongside an analysis of regionally specific effects (e.g., mass univariate voxelwise analysis underlying traditional statistical parametric mapping) and the two approaches should be seen as complementary. In the next sections we focus on specific advantages or insights gained from connectivity analyses.

\section{CONNECTIVITY ANALYSES DEMONSTRATE INCREASED SENSITIVITY TO DISEASE}

Many studies of neurological disease have reported significant effects in terms of network integration when none was found in terms of activation of individual nodes of the network. This phenomenon has been observed with many different connectivity methods, from some of the earliest applications in aging (Horwitz et al., 1986), Parkinson's disease (Grafton et al., 1994) and neurodevelopmental disorders (Horwitz et al., 1988) to more recent fMRI studies (below).

Rowe et al. (2007) combined fMRI and focal brain lesions in adults, to test the predictions of a model of prefrontal cortical function that had been developed from neuroimaging of healthy subjects (Sakai and Passingham, 2003, 2006). It was proposed that prefrontal cortex was essential to support task-set activations within task-specific non-prefrontal cortical regions in anticipation of future cognitive tasks. However, prefrontal lesions that included sites of activation in normal subjects did not diminish activation in surviving regions of the dorsal and ventral streams associated with anticipated future spatial and letter tasks. Could it really be that activation of prefrontal cortex in healthy volunteers was redundant? The authors then examined the correlations among surviving task-related brain regions during long delay intervals (Figure 1). Unlike DCM or SEM, no assumptions were required about the presence or direction of causal influences among these regions, nor the sources of perturbation of network activity. It was clear that the functional connectivity was impaired among surviving regions following prefrontal cortical lesions, even though the mean level of activation of each region during the task was not altered in patients. This illustrates that even a simple correlations method was sufficient to test the hypothesis regarding necessity of a region, and to identify group differences that were not apparent from the analysis of regional activations.

Many neurological disorders affect movement and action - how actions are chosen, learned, imagined, and executed. These "motor" processes depend on an extended motor system of cortical and subcortical regions, including motor and premotor cortex, prefrontal and parietal association cortex, striatum, thalamus, and cerebellum. The anatomical connections among these regions of the motor network have been extensively described in non-human primates (Kotter, 2004; Stephan et al., 2001), sufficient to build simple but plausible anatomical models related to action which can be supplemented by human anatomical connectivity estimates using diffusion weighted imaging (Johansen-Berg and Rushworth, 2009; Stephan et al., 2009b).

The function of this extended motor system is clearly relevant to understanding the common movement disorder Parkinson's disease (PD) and its treatment. Many studies have used mass univariate analyses of regional activation (e.g., Statistical Parametric Mapping of the fMRI response arising from focal neural activity) to compare movement related activations in PD "on" or "off" medication, against healthy adults (Hughes et al., 2010). These studies have identified abnormalities in the supplementary or pre-supplementary motor area (SMA) and lateral premotor cortex (PMC). However, the network function could not be inferred from differences in localized activations alone.

Whereas Rowe et al. (2007) examined a few specified connections, the correlations or covariances among multiple brain regions can be examined without prior specification of network nodes or structure. Orthogonal spatiotemporal patterns of activation may be identified by partial least squares (PLS) or principal components analysis (PCA) and related back to task demands or disease states (McIntosh et al., 2004; McIntosh and Lobaugh, 2004). This enables exploration but also hypothesis testing for complex networks. For example, PLS analysis of PET data has shown that to learn motor sequences as effectively as controls, patients with PD over-express the same fronto-parietal network that is associated with the task in healthy adults (Mentis et al., 2003). One cannot infer the structure of direct or indirect causal connections within this covarying network, or the temporal dynamics of the network from PET data. Nonetheless, the PLS was sufficient to test major hypotheses regarding efficiency versus additional recruitment of brain networks in PD. Relatively few studies of neurological disorders have so far used PLS/PCA for fMRI data. However, they may be more sensitive to group effects than mass-univariate methods (McIntosh and Lobaugh, 2004) and the lack of assumptions about the anatomy or architecture of affected networks is a potential advantage.

One of the earliest examples of network analysis of neuroimaging data was SEM of cortico-subcortical interactions in PD, using positron emission tomography (PET) before and after treatment by pallidotomy (Grafton et al., 1994). Grafton et al. (1994) used published anatomical and electrophysiological data (e.g., Alexander et al., 1990) to build an anatomical model, and then used SEM to identify differences in movement-related connectivity between groups, before and after therapeutic pallidotomy. Their path analysis again revealed significant differences in connectivity even when categorical differences in activity within regions was not observed between groups.

Grafton et al. (1994) also showed that treatment by pallidotomy attenuated thalamocortical connectivity, "downstream" from the lesion. This illustrates another recurrent finding, that connectivity changes may be remote from the lesion in complex networks. Indeed, in circuits with recurrent projections, the effects of a lesion may be seen in altered connectivity apparently "upstream" of the lesion. For Grafton et al. (1994), this occurred in SMA projections to the putamen, but it also occurs in SEM simulations of focal lesions in complex networks (Kim and Horwitz, 2009). 


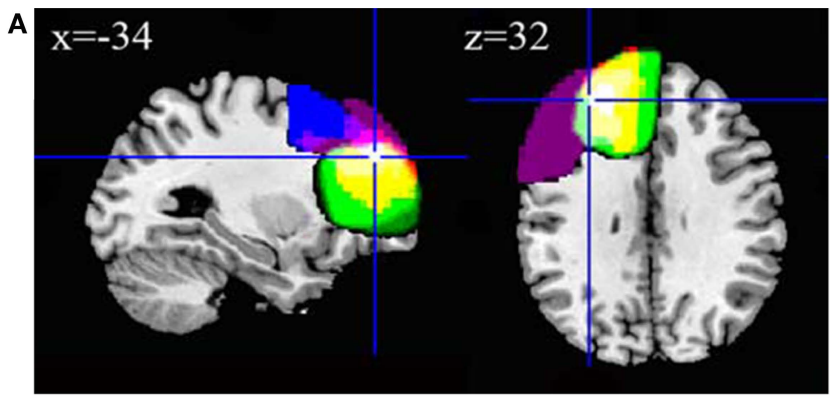

B 0.7

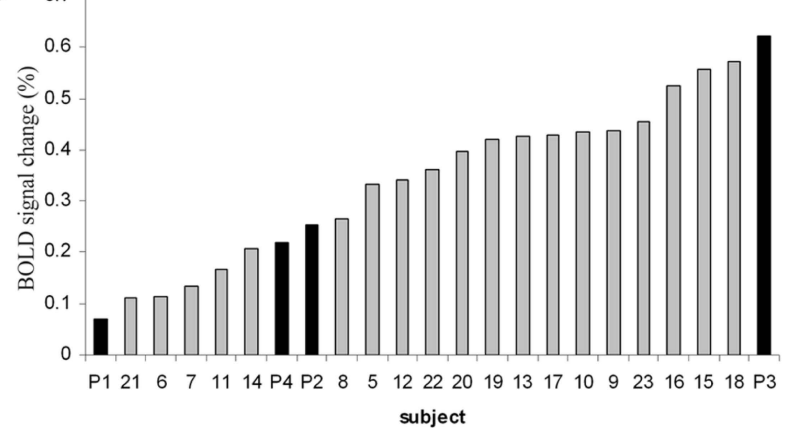

C

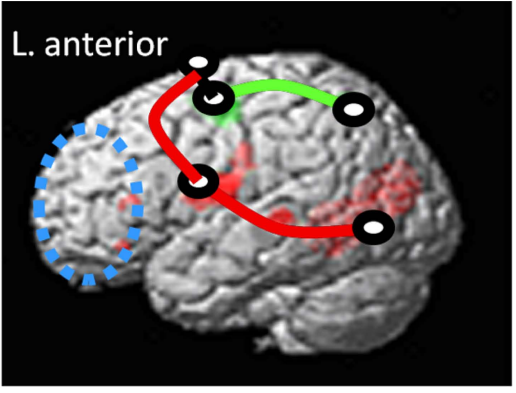

D

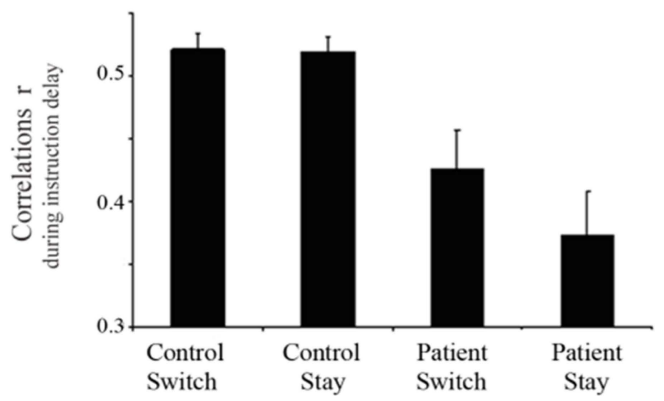

FIGURE 1 | During sustained task set, for future verbal or spatial working memory tasks, the lesions of left prefrontal cortex (A) made no significant difference to behavior or activations in surviving non-prefrontal cortex. For example, the estimates percent BOLD signal change in left inferior frontal gyrus (B) were not lower in four patients (black bars) compared with health controls (gray bars) However, the correlations among five surviving regions (C) associated with verbal set (red) or spatial set (green) were reduced in patients (D), especially when the same task set was repeated in subsequent trials (stay trials) (Rowe et al., 2007).
Rowe et al. (2002a,b) used SEM of fMRI to study simple finger movement and attention to action in Parkinson's disease. The SEM again used an anatomical model derived from human and animal data in the literature. The model also embodied psychophysiological interactions (Friston et al., 1997) in terms of modulatory influences of attention on inter-regional connectivity. Voxel-wise analysis revealed no significant group activation differences in prefrontal cortex or lateral premotor cortex. SMA activation was greater in patients than controls for simple movements, but less in patients for attention to action, consistent with previous fMRI studies (Catalan et al., 1999; Sabatini et al., 2000). SEM indicated that attention to action specifically enhanced connectivity from prefrontal cortex to SMA and premotor cortex (PMC) in healthy adults. However, the SEM also revealed that the modulatory effect of attention to action on connectivity disappeared in patients with PD. This was interpreted as a functional disconnection of the SMA (Dick et al., 1986). In other words, the SEM approach showed that the SMA was not inherently over- or under-active in Parkinson's disease, but instead it was no longer subject to appropriate modulation by prefrontal afferents.

One feature of the model used by Rowe et al. (2002a,b) was that the changes in connectivity might have arisen within either direct cortico-cortical connections or at some unspecified point in the cortico-striato-thalamo-cortical loops. For some readers, the ability to identify cortico-cortical connectivity at a systems level, without specifying intermediate paths, is an advantage. However, for others, the inadequacy of the models leaves unanswered important questions about the mechanisms of effect of PD on movement related networks.
Structural equation modeling has several other limitations (Buchel and Friston, 1997; Penny et al., 2004b; Kim et al., 2007). These include the inability to compare non-nested alternative network models (where one network is not a component of the other); the assumption of stationary of neural responses; severe restrictions on the complexity of models; and in some implementations an inflexible canonical hemodynamic response function. These disadvantages can be circumvented by DCM while retaining the hypothesis led investigation of the effects of $\mathrm{PD}$ on intrinsic network connectivity and context-dependent modulatory influences of connectivity (note that DCM still requires the same data, i.e., nodes, in all models, even if they are not nested).

Rowe et al. (2010) therefore revisited the effects of PD on an extended cortical motor network in the frontal lobe using DCM. Participants made simple hand movements that were either selfselected or externally specified (rather than attended to as in the earlier study). Again, voxel-wise analysis revealed no significant action-related or choice-related activation differences between PD and control subjects in the SMA or PMC. The DCM employed a Bayesian framework to identify subject-specific and region-specific neurovascular forward models; the effects of choice on connectivity; and estimated the model-evidence of each member of a large set of candidate models. DCM model selection procedures re-identified the same optimal model in young and old healthy controls, and were reliable across repetitions several weeks apart. In accordance with the earlier SEM study, there was contextual modulation of connectivity from the lateral prefrontal cortex to the pre-SMA (Figure 2). Given the commonalities between attention 

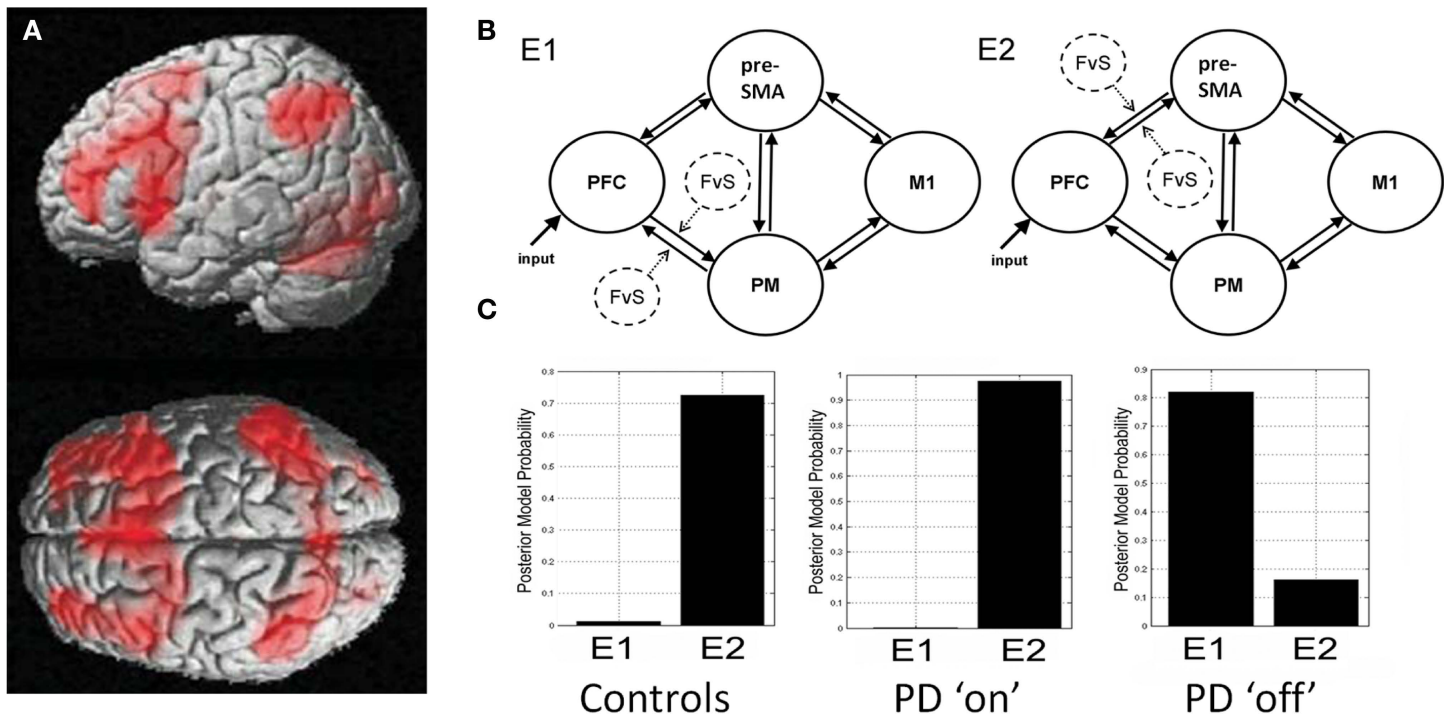

FIGURE 2 | (A) During manual action selection, there is activation of prefrontal cortex (PFC), pre-supplementary motor area (pre-SMA), lateral premotor cortex (PMC) and primary motor cortex (M1). These activations did not differ between patients with Parkinson's disease (PD) and control subjects, by voxel-wise group comparisons. (B) Dynamic causal modeling (DCM) was used to model the interactions among these regions. Forty-eight models were compared in all, differing in terms of anatomical connections, feed-forward versus feedback, and the connections which are subject to modulation by selection of action (FvS). The two leading models are shown in detail here (E1 and E2). (C) In healthy subjects, and patients on their dopaminergic medication, model E2 was more likely (by the posterior model probability, based on the free energy estimate of the log of modelevidence, adjusted for model complexity) in which the selection of action (FvS) was associated with greater connectivity of PFC to pre-SMA. When withdrawn from medication, to a relative "off" state, the connectivity pattern in PD patients changed to a state in which the selection of action was associated with greater connectivity between PFC and the PMC, model E1. This confirmed the hypothesis of a functional disconnection of the pre-SMA, and an enhanced role of the lateral PMC in action selection in PD. From Rowe et al. (2010). to action and attentional selection of action, it is notable that the two very different connectivity methods - SEM and DCM - led to similar conclusions.

Despite the lack of significant differences in local activations between PD and controls, the DCM revealed clear differences in group connectivity. Not only was DCM more sensitive than classical voxel-wise analysis to the effects of $\mathrm{PD}$, it was also sensitive to the connectivity changes associated with effective dopaminergic therapy (Rowe et al., 2010; cf Grafton et al., 1994). For patients in an "off" state after drug withdrawal, PD abolished the contextual modulation of prefrontal connectivity to the pre-SMA (self-selected versus specified actions), replaced by contextual modulation of connectivity from prefrontal cortex to lateral PMC. When patients were "on" after dopaminergic medication, the normal pattern of connectivity was restored.

Rowe et al. (2010) also showed that DCM was reproducible in health, at least in terms of the model selection procedures. However, there were significant posterior covariances among parameter estimates. These meant that individual parameters were not uniquely identifiable. The lack of unique identification means that estimated parameter values may correlate poorly across sessions even for the same tasks in the same subjects across sessions, making them unsuitable for use as dependent variables in statistical comparisons of groups. This is despite the excellent reliability of model selection procedures. For other tasks and models, DCM connectivity parameter estimates may be identifiable and therefore more reliable (Schuyler et al., 2009). In practice, we suggest that the major hypotheses regarding the effects of PD on motor network connectivity could be formulated in terms of model selection, as this was both reliable and sensitive.

The superior sensitivity of DCM to neurodegenerative disease, compared to analysis of regional activation, has also been observed for primary progressive aphasia (PPA), a subtype of frontotemporal dementia (Sonty et al., 2007). PPA is a structurally and cognitively restricted syndrome, with left perisylvian and temporal lobe atrophy and progressive language impairments, clearly suited to an hypothesis-led anatomically constrained approach like DCM. For a semantic task during fMRI, there were clear task-specific activations in the left inferior frontal gyrus (Broca's area) and posterior superior temporal gyrus (Wernicke's area) regions in both groups. Neither region's activity differed significantly between groups. However, despite the lack of differential activation within these regions, connectivity between Wernicke's and Broca's area was reduced in patients. The functional relevance of this connectivity difference is considered in the next section.

The previous studies of PD and PPA might have revealed changes in connectivity because either area or the connections between them are directly affected by the pathology. This direct effect of pathology in a network is not always the case. For example, Sharma et al. (2009) studied the imagination of movement following subcortical stroke. This was primarily motivated by the need to study the motor system in patients with weakness of actual movements. With the exception of primary motor cortex, healthy subjects trained to imagine hand movements demonstrated activation throughout the motor network, to a very similar degree as actual movements of 
the same rate. However, without actual movements, it is difficult to specify the times of imagined movements. This led Sharma et al. (2009) to use SEM of fMRI data, rather than DCM, adopting the assumptions of stationarity rather than dynamic networks and sacrificing model selection procedures in favor of an anatomical model based on the literature.

Following significant partial recovery from stroke, the regional activations in patients were not different from healthy control subjects (Sharma et al., 2009). However effective connectivity within the motor system remained abnormal, both for actual and imagined movements (Figure 3). An earlier study of stroke patients performing manual actions data had also shown functionally relevant changes in cortical connectivity using DCM rather than SEM (Grefkes et al., 2008). Importantly for Sharma et al. (2009) the cortical motor network model did not include the subcortical lesions.
Connectivity differences were concentrated on the interactions between prefrontal cortex, the premotor cortex and SMA, reminiscent of the abnormalities of frontal motor networks seen in PD. Thus, this study of stroke reiterates two findings: that connectivity analyses can be more sensitive to disease than analyses of regional activations, and that connectivity effects may be distant from a focal pathology.

\section{DISTRIBUTED (DIS-)CONNECTIVITY FOR DISTRIBUTED NEUROPATHOLOGY}

Neurodegenerative diseases often have characteristic anatomical distributions reflecting selective vulnerability of neurons (Braak and Braak, 1997; Braak et al., 2006). Even within frontotemporal dementia there are distinct behavioral and language clinical phenotypes characterized by different anatomical distributions
A Controls Motor Imagery
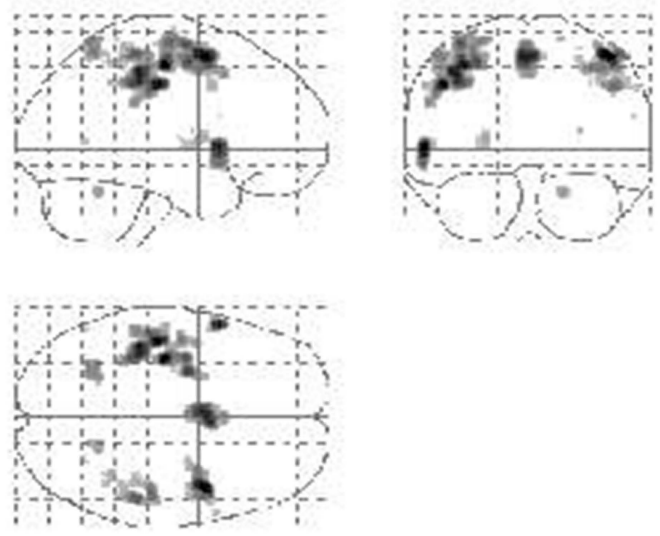

B

Stroke Motor Imagery
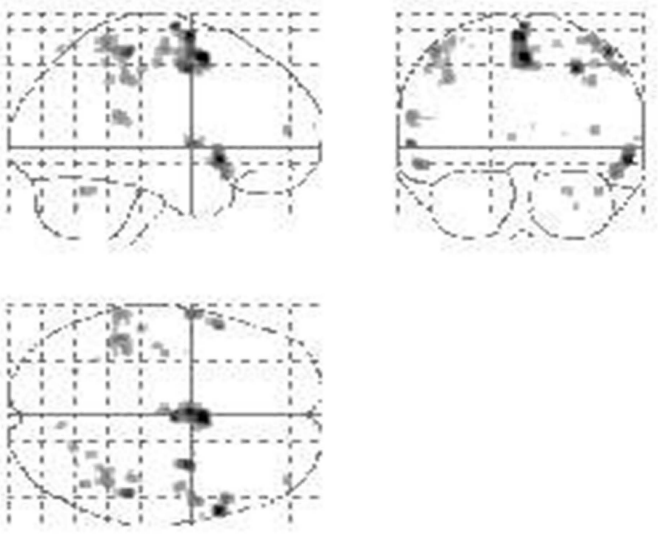

C
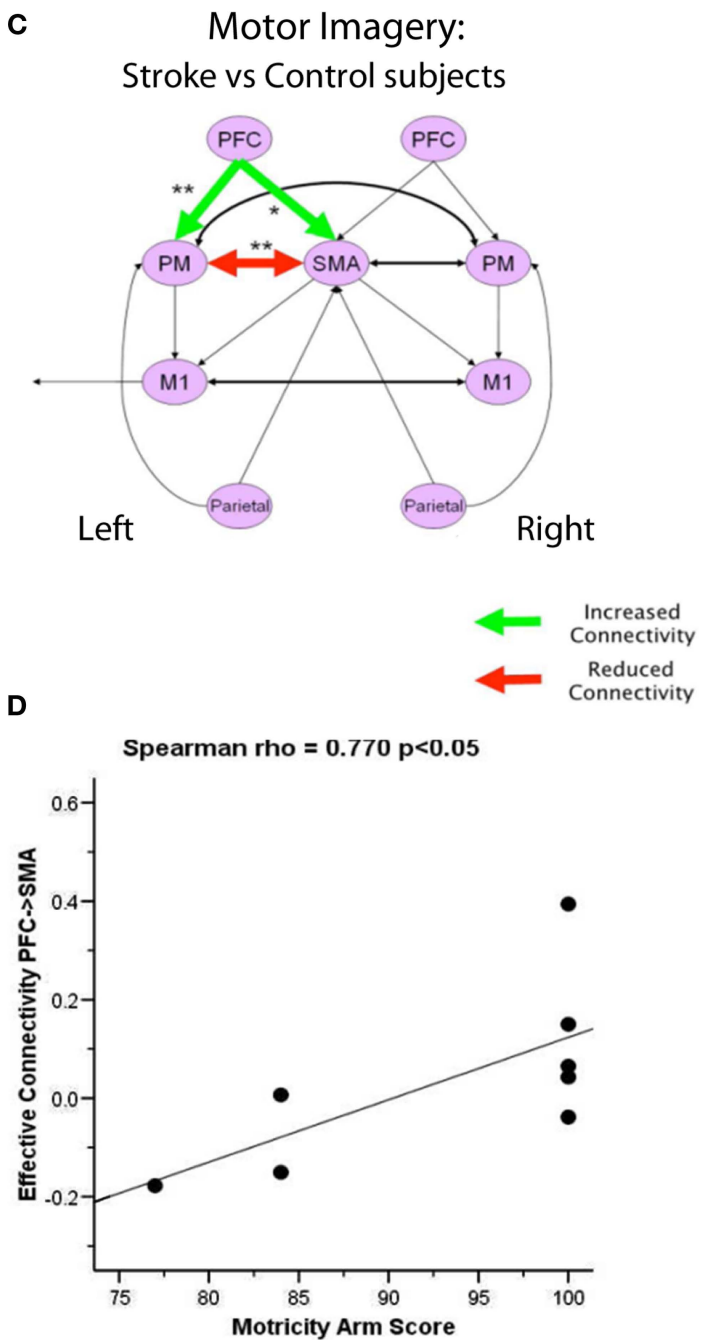

(C) even after substantial clinical recovery. Patients showed increased connectivity from left prefrontal cortex to the SMA and premotor cortex. Moreover, the connectivity path coefficient from right PFC to the SMA correlated with the function of the recovered arm (D). From Sharma et al. (2009). 
of disease burden (Pereira et al., 2009; Rohrer et al., 2010). How do these relate to distributed functional systems in the normal brain?

Progress in understanding the relationship between pathology, structural, and functional networks has been made in recent years by the integration of multiple imaging modalities. This stems from the recognition of a small set of functionally connected (coactivated) networks, conserved across spontaneous fluctuations in primate networks (Vincent et al., 2007); humans with impaired consciousness (Boly et al., 2008; Greicius et al., 2008); and during rest in awake humans (Beckmann et al., 2005; Damoiseaux et al., 2006; Fox and Raichle, 2007; Smith et al., 2009). One of these "resting state networks" (RSNs) is a "default mode" that is prominent during the interval between focused cognitive tasks in humans (Fox and Raichle, 2007; Buckner et al., 2008). The robustness of RSNs has attracted considerable interest to understand or predict the effects of distributed neuropathologies.

Resting state networks are particularly useful for studying severely impaired clinical populations. The advantage lies not only the avoidance of some practical difficulties such as training and differential performance on cognitive tasks; long scanning sessions; and dependence on prior specification of anatomical model connectivity. RSNs also enable the comparison across multiple clinical conditions on multiple brain networks.

In two seminal papers, Seeley et al. (2009; Zhou et al., 2010) analyzed RSNs in several heterogeneous neurodegenerative diseases, including Alzheimer's and frontotemporal dementia. The disorders varied in their pathology and the distribution of disease burden across cortical and subcortical regions. There were corresponding disease-specific changes in RSNs that were robust enough to enable classification of disease (Zhou et al., 2010). The abnormal RSNs were in accordance with functional deficits typical of each disorder with evidence that the RSNs are selectively vulnerable to neuropathology, measured by focal atrophy (Greicius, 2008; Supekar et al., 2008; Seeley et al., 2009).

The correspondence of structural and functional connectivities does not imply that the progression of neuropathology through the networks depends on the same structural interconnections. This might be the case, especially for diseases with prior-like behavior of aggregating proteins, including PD and Alzhiemer's disease (Soto and Estrada, 2008; Angot and Brundin, 2009; Morales et al., 2010). However, it may also result from developmentally or environmentally determined predispositions to neuropathology in functionally related neurons, even when distributed widely across the RSN and in the absence of direct connection. In addition, artifactual sources of functional connectivity must also be considered, such as aliased cardiorespiratory signals or gross head movements (Birn et al., 2006) which may differ systematically between patient groups. Fortunately, where these sources of noise are measured, ICA can be used to identify and account for their otherwise misleading contributions to disease-specific RSNs (Beckmann et al., 2005).

Interestingly, disease-specific changes in RSN functional connectivity can occur in the absence of known direct structural connectivity (Damoiseaux and Greicius, 2009; Honey et al., 2009). The presence of indirect structural connections is one possible explanation. However, recall that the task-based changes in effective connectivity in the previous section (Rowe et al., 2007; Sharma et al.,
2009) occurred in parts of a network that were not directly affected by the lesion (tumor resection and stroke respectively). It is also possible that neurochemical depletion rather than focal atrophy or white matter disease may cause differences in connectivity (Rowe et al., 2002b, 2008b,c; Palmer et al., 2009; Wu et al., 2009).

\section{CONNECTIVITY CHANGES CAN BE FUNCTIONALLY RELEVANT}

In this section, we consider the functional relevance of changes in effective and functional connectivity. We have already seen that in primary progressive aphasia, DCM measures of connectivity were abnormal even when regional activations were not. Sonty et al. (2007) went on to show that the abnormality of DCM connectivity correlated with cognitive tests of disease severity. Specifically, in patients but not controls, the connectivity between Wernicke's and Broca's areas correlated with performance on a semantic task but not a lexical task. Thus, the change in connectivity reflected the functionally relevant cognitive defects that characterize the PPA syndrome.

It is interesting to compare the task-specific abnormal frontotemporal connectivity in PPA (Sonty et al., 2007) with the abnormal RSN in other forms of frontotemporal dementia (Seeley et al., 2009). The former provides a direct connectivity-based mechanism of the cognitive clinicopathological correlations within PPA. In contrast, the latter study helps one begin to understand the basis of selective vulnerability of distributed neural networks to different pathological processes between neurodegenerative syndromes. This difference reinforces the suggestion (Section Which Method of Connectivity Should One Choose?) that the method to study connectivity should be chosen according to the hypothesis to be tested and type of inferences that are sought.

There is further evidence of the functional relevance of differences in connectivity. Following subcortical stroke, prefrontal interactions with premotor cortex and SMA were abnormal even though none of the regions was directly affected by the stroke or differentially activated during the task (Sharma et al., 2009). Moreover, the degree of connectivity (SEM path coefficients) in the frontal lobe motor network correlated with functional indices of recovery (Figure 3). For example the SEM path coefficient for prefrontal cortex to SMA during motor imagery correlated with the motricity index of arm function.

In the studies of PD outlined above, the analyses of effective connectivity have been shown to be sensitive to conventional effective therapies. Both pallidotomy and L-DOPA medication lead to significant differences in effective connectivity using SEM and DCM respectively (Grafton et al., 1994; Rowe et al., 2010). Interestingly, not all PD related changes in connectivity are normalized by l-DOPA (Palmer et al., 2009), consistent with noradrenergic and serotonergic contributions to cognitive dysfunction in PD (Marsh et al., 2009; Rodriguez-Oroz et al., 2009).

Even the RSN abnormalities may be directly related to functional ability, over and above their indexing of network dysfunction. For example, although RSNs are recorded at "rest," the properties of these networks are functionally relevant to performance on specific cognitive tasks (Kelly et al., 2008; Hayden et al., 2009). Despite the evidence presented so far of the sensitivity, reproducibility, and functional relevance of connectivity measures, there are serious problems and caveats to be considered. We turn to these in the next section. 


\section{LIMITATIONS, COUNTER INTUITIVE RESULTS, AND NAIVE EXPECTATIONS}

The analysis of effective connectivity and the terminology used in neuroimaging are inspired by neurophysiology, neuropsychology, and a computational analysis of neuronal interactions (Gerstein and Perkel, 1969; Aertsen et al., 1989; Friston, 2007). There are attempts to explicitly bridge between cellular and whole brain methods (Chawla et al., 1999; Riera et al., 2006), and the conceptual framework from cellular models has proven useful in imaging analysis. However, it should be borne in mind that there is much that we do not know about the translation between neuronal activity and the PET and BOLD signals (Logothetis and Pfeuffer, 2004; Goense and Logothetis, 2008). Pre- and post synaptic functions of neurotransmission as well as spike rates are relevant to the generation of a vascular response, via complex and incompletely characterized coupling mechanisms. The BOLD signal underlying most of the results discussed here is therefore only an indirect measure of neuronal function, and this may be an important caveat for neuroimaging applications to specific diseases or treatments. More generally, it means that the connectivity measures in, say, DCM, PPIs, or SEM, cannot therefore be interpreted in terms of single excitatory or inhibitory synapses.

This limitation partly reflects the fact that current structural models in DCM or SEM are specified at a systems level, rather than the mono- or poly-synaptic connections of direct, indirect, or parallel connections between regions. In addition, current fMRI provides a single state representation for each node (voxel or region) in which the functions of all types of neuron and all types of synapse in a voxel are collapsed to a single value at any given timepoint. A richer descriptive framework with multi-state representations per region is beginning to emerge, at least for M/EEG data (Chen et al., 2008, 2009; Marreiros et al., 2008). Meanwhile, the uncertainty over the physical basis of connectivity in, say, PPI-GLMs, may explain some results that otherwise appear contradictory (Passamonti et al., 2009).

Confounding factors and correlations among model parameters must also be considered (Deneux and Faugeras, 2006; Rowe et al., 2010). For example, Rowe et al. (2010) found that DCM model comparison was very reliable, but posterior covariances among parameters meant that individual model parameters were no longer uniquely identifiable, and were therefore not reliable. This would inflate the false negative rate of group or drug comparisons. In addition, correlations among parameters can lead to the counterintuitive result that the arithmetic mean of parameters for a group may differ markedly (even in terms of sign) from the precision weighted Bayesian "averaging" of parameters across the group. If using DCM, a consistent, principled and prior specification of the analysis protocol must therefore be developed to reduce bias (Stephan et al., 2010). Alternative approaches to causal dynamic models (related conceptually to DCM and GCM) have also been developed (Smith et al., 2010a) with a view to reliable parameter estimation, but it is too soon to comment on their application to clinical populations.

Connectivity approaches like DCM have further highlighted the problems of simplistic interpretations or inconsistent applications of "top-down" and "bottom-up" processes in terms of feed-back and feed-forward connections (Mechelli et al., 2003; Penny et al., 2004a; Garrido et al., 2007). For example, "top-down” cognitive control may manifest as changes in feed-forward connections (Rowe et al., 2008a). This was identified using DCM, as modality specific changes in connectivity from prestriate to parietal and temporal cortex during a task in which cognitive set varied between visual and spatial domains (Figure 4). Fortunately, evidence-based model selection procedures in DCM can be used to determine whether the functional anatomical network has significant feed-forward and feed-back connections, even where these do not map directly to cognitive psychological constructs such as "top-down" and "bottom-up" control (Mechelli et al., 2003; Rowe et al., 2008a).

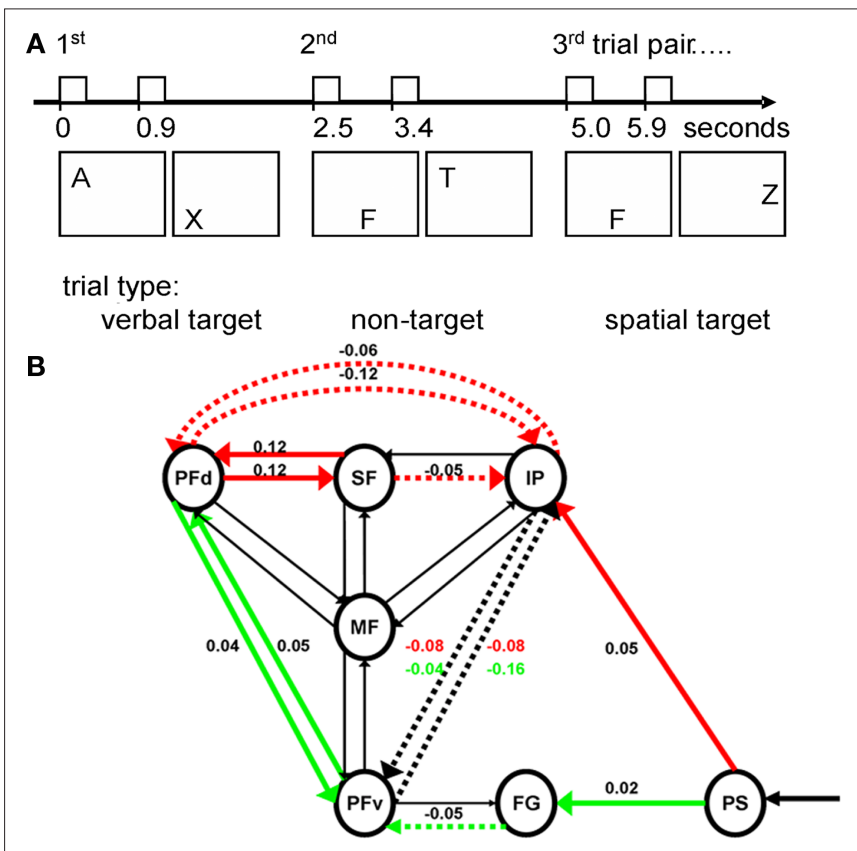

FIGURE 4 | (A) During a two-modality continuous performance task, subjects monitored a letter stream for successive verbal targets ( $A$ then $X$ ) or successive spatial targets ( 3 then 6 o'clock positions). Three correct targets within a modality were rewarded. Reward expectations lead to a graduated bias toward verbal or spatial cognitive sets, according to the recent history of spatial versus verbal targets. (B) The effects of this "top-down" modulation from cognitive set were studied using dynamic causal modeling of fMRI data. The figure shows modulatory (bilinear) effects representing psychophysiological interactions in the most likely causal model (selected by Bayesian model comparison). This model included the medial frontal (MF) cortex, the dorsal (PFd) and ventral (PFv) lateral prefrontal cortex, the superior frontal sulcus (SF), the intraparietal cortex (IP), the fusiform gyrus (FG), and the prestriate cortex (PS), with intrinsic connections indicated by the presence of arrows (of any color). Values are time constants $(\mathrm{Hz})$ for the modulatory influences of task bias for which the group posterior mean was positive (solid lines) or negative (dashed lines) for verbal bias (thick green), spatial bias (thick red), or both (thick black). These modulatory effects have strong evidence that they are non-zero, confirmed by post hoc t-tests. The "top-down" modulation of task set resulting from higher reward expectations was associated not only with changing connectivity of the lateral prefrontal cortical regions, but also the feed-forward connections from pre-striate cortex. Moreover, the feed-forward connections were enhanced to parietal cortex with spatial task set bias, and to temporal cortex with verbal task set bias. This illustrates that domain specific "top-down" control is not restricted to changes in feedback connections from higher cortical areas, but is also manifest by changes in feed-forward connectivity. From Rowe et al. (2008a). 
Like mass-univariate modeling of regional activations, connectivity methods are also prone to technical, statistical, and inferential problems arising from clinical group differences in confounds, sources of variance, and performance. Important confounding factors to consider are motion artifacts (which are often greater in clinical populations), cardiorespiratory signals (altered by anxiety or medications such as beta-blockers), age, and systematic differences in the hemodynamic response function (Iannetti and Wise, 2007). A patient population will often have additional sources of variance due to the heterogeneity of disease phenotype, duration, severity and response to treatments, or differences in artifacts as above. Group level inferences of connectivity should attempt to accommodate or adjust for these inequalities of variance, whether at the first level adjustment of the data to include in time-series analysis (Glover et al., 2000) or second level random effects models (Stephan et al., 2009a). Otherwise, outlying subjects or mixed generative models within a group may compromise group comparisons.

The problem of differential sources of variance between groups can have other counterintuitive consequences. Using simulated fMRI data, Kim and Horwitz (2009) compared SEM network connectivity in controls with patients following a focal network lesion. Surprisingly, goodness of fit (GFI) estimates were better for the patients, yet patients showed significantly larger error variances throughout the network. The better GFI values had resulted from better modeling of error variance on each node rather than the values of inter-regional connectivity. High GFI values for models of patient data might therefore arise when regional activations are affected mostly by their noise inputs. Understanding, minimizing, and controlling for group differences in variance is therefore essential.

We have presented several cases in which connectivity analysis revealed effects of disease or treatment when the analysis of regional activations did not. This may reflect a true higher sensitivity to the neurophysiological effects of disease or treatment, although one cannot rule out a publication bias and investigator bias toward connectivity analysis when traditional approaches "fail." Multivariate methods like PLS or PCA may have intrinsically higher power than univariate analyses. This is supported by simulated and clinical data (McIntosh et al., 2004; Asllani et al., 2008; Habeck et al., 2008), but methods differ widely (Smith et al., 2010b) and increased sensitivity to network changes is generally at the expense of information about the organization of interactions within that network. For the analysis of effective connectivity, within theoretically motivated and anatomically constrained networks, it does not seem that methods like DCM have inherently higher power to detect underlying effects. For example, in a fMRI study of face emotion processing in two populations (Goulden et al., 2010) the number needed to achieve reasonable power $(>0.7)$ to detect large effects $(0.8)$ in DCM parameters was approximately 20 subjects. This estimate is similar to the number indicated for typical mass-univariate analysis of voxel-wise activations (Desmond and Glover, 2002; Murphy and Garavan, 2004; Mumford and Nichols, 2008).

Performance difference between groups must also be considered. If patients do not perform a task, then there is an inherent ambiguity to differences in neuroimaging data (Price and Friston, 1999; Price et al., 2006). This ambiguity is often not resolved in clinical studies, although the use of parametric modulations of task or performance indices can be used to try to circumvent the difficulty in interpreting categorical differences. Connectivity studies have the added problem of interpreting connectivity differences if there are differences in regional activations. In the extreme case, if a disease reduces a region to noise only, or removes a region altogether, then changes in its connectivity are not meaningful. However, as we have seen, diseases can cause significant changes in connectivity among surviving regions with normal activation (Rowe et al., 2007, 2008b; Sonty et al., 2007; Sharma et al., 2009).

\section{CONCLUDING REMARKS}

We have shown that the analysis of brain connectivity has much to contribute to understanding the consequences and mechanisms of neurological disease, and complements other neuroimaging methods. It is sensitive to disease and therapies, and relates to functional loss or recovery, following a wide variety of focal and distributed pathologies.

Colleagues have sometimes asked "I have this experiment that hasn't really worked so should I do connectivity analysis?” or "A reviewer has asked me to do a connectivity analysis, but isn't clear why. What should I do?" There are clearly cases in which the standard analysis of regional activations has yielded minimal results, despite functionally relevant difference in connectivity. The investment of time and resources in undertaking the connectivity analysis is certainly worthwhile. However, if a planned study of regional activations has failed in its main aims, it is best to begin by revisiting the hypotheses that led to the experiment and considering potential causes and confounds underlying negative results. Many of the same problems will undermine a connectivity analysis as well.

Despite the yield from the analysis of connectivity, these methods should be motivated by specific hypotheses. Moreover, it is often better to plan a new experiment with the intention of connectivity analysis in mind. This may require modifications to the range of stimulus conditions, or the reformulation of specific hypotheses, e.g., in terms of model selection rather than non-zero path parameters. Bear in mind that an experimental design may not be suitable for some connectivity methods, even if optimal for other forms of analysis (Henson, 2007). As for other neuroimaging studies, it is important to specify in advance the protocols for construction of models, optimization, and inference, in order to reduce biases in inference and estimation of the presence or size of connectivity effects (Kraft, 2008; Kriegeskorte et al., 2009; Vul et al., 2009).

Through this article, we hope to encourage researchers to engage in formal analyses of brain connectivity. These methods are practicable, insightful, and exciting. Each of the methods I have discussed here is supported by readily available freeware or commercial software (see Table 1), with discussion forums and on-line support to supplement published methods. Although the methods are evolving rapidly, we have no doubt that they will continue to make an essential contribution to our understanding of neurological and neuropsychiatric disease.

\section{ACKNOWLEDGMENTS}

James B. Rowe is supported by the Wellcome Trust (088324) and the Medical Research Council (Cognition and Brain Sciences Unit). 


\section{REFERENCES}

Aertsen, A. M., Gerstein, G. L., Habib, M. K., and Palm, G. (1989). Dynamics of neuronal firing correlation: modulation of "effective connectivity." $J$. Neurophysiol. 61, 900-917.

Alexander, G. E., Crutcher, M. D., and DeLong, M. R. (1990). Basal gangliathalamocortical circuits: parallel substrates for motor, oculomotor, "prefrontal" and "limbic" functions. Prog. Brain Res. 85, 119-146.

Angot, E., and Brundin, P. (2009). Dissecting the potential molecular mechanisms underlying alphasynuclein cell-to-cell transfer in Parkinson's disease. Parkinsonism Relat. Disord. 15(Suppl. 3), S143-S147.

Asllani, I., Habeck, C., Scarmeas, N., Borogovac, A., Brown, T.R., and Stern, Y. (2008). Multivariate and univariate analysis of continuous arterial spin labeling perfusion MRI in Alzheimer's disease. J. Cereb. Blood Flow Metab. 28, 725-736.

Beckmann, C. F., DeLuca, M., Devlin, J. T., and Smith, S.M. (2005). Investigations into resting-state connectivity using independent component analysis. Philos. Trans. R. Soc. Lond. B Biol. Sci. 360, 1001-1013.

Birn, R. M., Diamond, J. B., Smith, M. A., and Bandettini, P.A. (2006).Separating respiratory-variation-related fluctuations from neuronal-activity-related fluctuations in fMRI. Neuroimage 31, 1536-1548.

Boly, M., Phillips, C., Tshibanda, L., Vanhaudenhuyse, A., Schabus, M., Dang-Vu, T. T., Moonen, G., Hustinx, R., Maquet, P., and Laureys, S. (2008). Intrinsic brain activity in altered states of consciousness: how conscious is the default mode of brain function? Ann N. Y. Acad. Sci. 1129, 119-129.

Braak, H., Bohl, J. R., Muller, C. M., Rub, U., de Vos, R. A., and Del Tredici, K. (2006). Stanley Fahn Lecture 2005: the staging procedure for the inclusion body pathology associated with sporadic Parkinson's disease reconsidered. Mov. Disord. 21, 2042-2051.

Braak, H., and Braak, E. (1997). Diagnostic criteria for neuropathologic assessment of Alzheimer's disease. Neurobiol. Aging 18, S85-S88.

Buchel, C., and Friston, K. (2000). Assessing interactions among neuronal systems using functional neuroimaging. Neural. Netw. 13, 871-882.

Buchel, C., and Friston, K. J. (1997). Modulation of connectivity in visual pathways by attention: cortical interactions evaluated with structural equation modelling and fMRI. Cereb. Cortex 7, 768-778.

Buckner, R. L., Andrews-Hanna, J. R., and Schacter, D. L. (2008). The brain's default network: anatomy, function, and relevance to disease. Ann. N. Y. Acad. Sci. 1124, 1-38.

Catalan, M. J., Ishii, K., Honda, M. Samii, A., and Hallett, M. (1999). A PET study of sequential finger movements of varying length in patients with Parkinson's disease. Brain 122, 483-495.

Chawla, D., Lumer, E. D., and Friston, K. J. (1999). The relationship between synchronization among neuronal populations and their mean activity levels. Neural. Comput. 11, 1389-1411.

Chen, C. C., Henson, R. N., Stephan, K. E., Kilner, J. M., and Friston, K. J. (2009). Forward and backward connections in the brain: a DCM study of functional asymmetries. Neuroimage 45 , 453-462.

Chen, C. C., Kiebel, S. J., and Friston, K. J. (2008). Dynamic causal modelling of induced responses. Neuroimage 41 , 1293-1312.

Cole, D. M., Smith, S. M., and Beckmann, C.F. (2010). Advances and pitfalls in the analysis and interpretation of restingstate FMRI data. Front. Syst. Neurosci. 4:8. doi: 10.3389/fnsys.2010.00008.

Damoiseaux, J. S., and Greicius, M. D. (2009). Greater than the sum of its parts: a review of studies combining structural connectivity and restingstate functional connectivity. Brain Struct. Funct. 213, 525-533.

Damoiseaux, J. S., Rombouts, S. A., Barkhof, F., Scheltens, P., Stam, C. J., Smith, S. M., and Beckmann, C. F. (2006). Consistent resting-state networks across healthy subjects. Proc. Natl. Acad. Sci. U.S.A. 103, 13848-13853.

Daunizeau, J., Friston, K. J., and Kiebel, S. J. (2009). Variational Bayesian identification and prediction of stochastic nonlinear dynamic causal models. Physica D 238, 2089-2118.

Deneux, T., and Faugeras, O. (2006). Using nonlinear models in fMRI data analysis: model selection and activation detection. Neuroimage 32, 1669-1689.

Desmond, J. E., and Glover, G. H. (2002). Estimating sample size in functional MRI (fMRI) neuroimaging studies: statistical power analyses. J. Neurosci. Methods 118, 115-128.

Dick, J. P., Benecke, R., Rothwell, J. C., Day, B. L., and Marsden, C. D. (1986) Simple and complex movements in a patient with infarction of the right supplementary motor area. Mov. Disord. 1, 255-266.

Fox, M. D., and Raichle, M. E. (2007). Spontaneous fluctuations in brain activity observed with functional magnetic resonance imaging. Nat. Rev. Neurosci. 8, 700-711.

Friston, K. (2007). "Functional integration," in Statistical Parametric Mapping, eds K. Friston, J. Ashburner, S. Kiebel, T. Nichols and W. Penny (London: Elsevier), 471-491.

Friston, K. (2009). Dynamic causal modeling and Granger causality comments on: the identification of interacting networks in the brain using fMRI: model selection, causality and deconvolution. Neuroimage. doi: 10.1016/j. neuroimage.2009.1009.1031

Friston, K. J., Buechel, C., Fink, G. R., Morris, J., Rolls, E., and Dolan, R. J. (1997). Psychophysiological and modulatory interactions in neuroimaging. Neuroimage 6, 218-229.

Friston, K. J., Harrison, L., and Penny, W. (2003). Dynamic causal modelling. Neuroimage 19, 1273-1302.

Garrido, M. I., Kilner, J. M., Kiebel, S. J., and Friston, K. J. (2007). Evoked brain responses are generated by feedback loops. Proc. Natl. Acad. Sci. U.S.A. 104, 20961-20966.

Gerstein, G. L., and Perkel, D. H. (1969). Simultaneously recorded trains of action potentials: analysis and functional interpretation. Science 164 828-830.

Glover, G.H., Li, T.Q., and Ress, D. (2000). Image-based method for retrospective correction of physiological motion effects in fMRI: RETROICOR. Magn Reson. Med. 44, 162-167.

Goense, J. B., and Logothetis, N. K. (2008) Neurophysiology of the BOLD fMRI signal in awake monkeys. Curr. Biol. $18,631-640$.

Goulden, N., McKie, S., Suckling, J., Williams, S. R., Anderson, I. M. Deakin, J. F., and Elliott, R. (2010). A comparison of permutation and parametric testing for between group effective connectivity differences using DCM. Neuroimage 50, 509-515.

Grafton, S., Sutton, J., Couldwell, W., Lew, M., and Waters, C. (1994). Network analysis of motor system connectivity in Parkinson's disease: modualtion of thalamocortical interactions after pallidotomy. Hum. Brain Mapp. 2 , 45-55.

Grefkes, C., Nowak, D. A., Eickhoff, S. B. Dafotakis, M., Kust, J., Karbe, H., and Fink, G. R. (2008). Cortical connectivity after subcortical stroke assessed with functional magnetic resonance imaging. Ann. Neurol. 63, 236-246.

Greicius, M. (2008). Resting-state functional connectivity in neuropsychiatric disorders. Curr. Opin. Neurol. 21 , 424-430.

Greicius, M. D., Kiviniemi, V., Tervonen, O., Vainionpaa, V., Alahuhta, S., Reiss, A. L., and Menon, V. (2008). Persistent default-mode network connectivity during light sedation. Hum. Brain Mapp. 29, 839-847.

Habeck, C., Foster, N. L., Perneczky, R., Kurz, A., Alexopoulos, P., Koeppe, R.
A., Drzezga, A., and Stern, Y. (2008). Multivariate and univariate neuroimaging biomarkers of Alzheimer's disease. Neuroimage 40, 1503-1515.

Hayden, B. Y., Smith, D. V., and Platt, M. L. (2009). Electrophysiological correlates of default-mode processing in macaque posterior cingulate cortex. Proc. Natl. Acad. Sci. U.S.A. 106 5948-5953.

Henson, R. (2007). "Efficient experimental design for fMRI," in Statistical Parametric Mapping, eds K. Friston, J. Ashburner, S. Kiebel, T. Nichols and W. Penny (London: Elsevier), 193-210.

Honey, G. D., Fu, C. H., Kim, J., Brammer, M. J., Croudace, T. J., Suckling, J., Pich, E. M., Williams, S. C., and Bullmore, E. T. (2002). Effects of verbal working memory load on corticocortical connectivity modeled by path analysis of functional magnetic resonance imaging data. Neuroimage 17, 573-582.

Honey, C. J., Sporns, O., Cammoun, L., Gigandet, X., Thiran, J. P., Meuli, R., and Hagmann, P. (2009). Predicting human resting-state functional connectivity from structural connectivity. Proc. Natl. Acad. Sci. U.S.A. 106, 2035-2040.

Horwitz, B. (2003). The elusive concept of brain connectivity. Neuroimage 19, 466-470.

Horwitz, B., Duara, R., and Rapoport, S. I. (1986). Age differences in intercorrelations between regional cerebral metabolic rates for glucose. Ann. Neurol. 19, 60-67.

Horwitz, B., McIntosh, A. R., Haxby, J. V., Furey, M., Salerno, J. A., Schapiro, M. B., Rapoport, S. I., and Grady, C. L. (1995). Network analysis of PET-mapped visual pathways in Alzheimer type dementia. Neuroreport 6, 2287-2292

Horwitz, B., Rumsey, J. M., Grady, C. L., and Rapoport, S. I. (1988). The cerebral metabolic landscape in autism. Intercorrelations of regional glucose utilization. Arch. Neurol. 45, 749-755.

Hughes, L. E., Barker, R. A., Owen, A. M., and Rowe, J. B. (2010). Parkinson's disease and healthy aging: Independent and interacting effects on action selection. Hum. Brain Mapp. doi: 10.1002 hbm.20979.

Iannetti, G. D., and Wise, R. G. (2007). BOLD functional MRI in disease and pharmacological studies: room for improvement? Magn. Reson. Imaging 25, 978-988.

Johansen-Berg, H., and Rushworth, M. F. (2009). Using diffusion imaging to study human connectional anatomy. Annu. Rev. Neurosci. 32, 75-94.

Kelly, A. M., Uddin, L. Q., Biswal, B. B., Castellanos, F. X., and Milham, 
M. P. (2008). Competition between functional brain networks mediates behavioral variability. Neuroimage 39, 527-537.

Kim, J., and Horwitz, B. (2009). How well does structural equation modeling reveal abnormal brain anatomical connections? An fMRI simulation study. Neuroimage 45, 1190-1198.

Kim, J., Zhu, W., Chang, L., Bentler, P. M., and Ernst, T. (2007). Unified structural equation modeling approach for the analysis of multisubject, multivariate functional MRI data. Hum. Brain Mapp. 28, 85-93.

Kotter, R. (2004). Online retrieval, processing, and visualization of primate connectivity data from the CoCoMac database. Neuroinformatics 2, 127-144.

Kraft, P. (2008). Curses - winner's and otherwise - in genetic epidemiology. Epidemiology 19, 649-651; discussion 657-648.

Kriegeskorte, N., Simmons, W. K., Bellgowan, P. S., and Baker, C. I. (2009). Circular analysis in systems neuroscience: the dangers of double dipping. Nat. Neurosci. 12, 535-540.

Lee, L., Friston, K., and Horwitz, B. (2006). Large-scale neural models and dynamic causal modelling. Neuroimage 30, 1243-1254.

Logothetis, N. K., and Pfeuffer, J. (2004). On the nature of the BOLD fMRI contrast mechanism. Magn. Reson. Imaging 22, 1517-1531.

Marreiros, A. C., Kiebel, S. J., and Friston, K. J. (2008). Dynamic causal modelling for fMRI: a two-state model. Neuroimage 39, 269-278.

Marsh, L., Biglan, K., Gerstenhaber, M., and Williams, J. R. (2009). Atomoxetine for the treatment of executive dysfunction in Parkinson's disease: a pilot open-label study. Mov. Disord. 24, 277-282.

McIntosh, A., and Gonzalez-Lima, F. (1994). Structural equation modeling and its application to network analysis in functional brain imaging. Hum. Brain Mapp. 2, 2-22.

McIntosh, A. R., Bookstein, F. L., Haxby, J. V., and Grady, C. L. (1996). Spatial pattern analysis of functional brain images using partial least squares. Neuroimage 3, 143-157.

McIntosh,A. R., Chau, W.K., and Protzner, A. B. (2004). Spatiotemporal analysis of event-related fMRI data using partial least squares. Neuroimage 23, 764-775.

McIntosh, A. R., Grady, C. L., Ungerleider, L. G., Haxby, J. V., Rapoport, S. I., and Horwitz, B. (1994). Network analysis of cortical visual pathways mapped with PET. J. Neurosci. 14, 655-666.
McIntosh, A. R., and Lobaugh, N. J. (2004). Partial least squares analysis of neuroimaging data: applications and advances. Neuroimage 23(Suppl. 1), S250-S263.

McKeown, M. J., Makeig, S., Brown, G. G., Jung, T. P., Kindermann, S. S., Bell, A. J., and Sejnowski, T. J. (1998). Analysis of fMRI data by blind separation into independent spatial components. Hum. Brain Mapp. 6, 160-188.

Mechelli,A., Price, C. J., Noppeney, U., and Friston, K. J. (2003). A dynamic causal modeling study on category effects: bottom-up or top-down mediation? J. Cogn. Neurosci. 15, 925-934.

Mentis, M. J., Dhawan, V., Feigin, A., Delalot, D., Zgaljardic, D., Edwards, C., and Eidelberg, D. (2003). Early stage Parkinson's disease patients and normal volunteers: comparative mechanisms of sequence learning. Hum. Brain Mapp. 20, 246-258.

Morales, R., Estrada, L. D., Diaz-Espinoza, R., Morales-Scheihing, D., Jara, M. C., Castilla, J., and Soto, C. (2010). Molecular cross talk between misfolded proteins in animal models of Alzheimer's and prion diseases. J. Neurosci. 30, 4528-4535.

Mort, D. J., Malhotra, P., Mannan, S. K., Rorden, C., Pambakian, A., Kennard, C., and Husain, M. (2003). The anatomy of visual neglect. Brain 126, 1986-1997.

Mumford, J. A., and Nichols, T. E. (2008). Power calculation for group fMRI studies accounting for arbitrary design and temporal autocorrelation. Neuroimage 39, 261-268.

Murphy, K., and Garavan, H. (2004). An empirical investigation into the number of subjects required for an event-related fMRI study. Neuroimage 22, 879-885.

Palmer, S. J., Eigenraam, L., Hoque, T., McCaig, R. G., Troiano, A., and McKeown, M. J. (2009). Levodopasensitive, dynamic changes in effective connectivity during simultaneous movements in Parkinson's disease. Neuroscience 158, 693-704.

Passamonti, L., Rowe, J. B., Ewbank, M., Hampshire, A., Keane, J., and Calder, A. J. (2008). Connectivity from the ventral anterior cingulate to the amygdala is modulated by appetitive motivation in response to facial signals of aggression. Neuroimage 43, 562-570.

Passamonti, L., Rowe, J. B., Schwarzbauer, C., Ewbank, M. P., von dem Hagen, E., and Calder, A. J. (2009). Personality predicts the brain's response to viewing appetizing foods: the neural basis of a risk factor for overeating. J. Neurosci. 29, 43-51.
Penny, W. D., Stephan, K. E., Mechelli, A., and Friston, K. J. (2004a). Comparing dynamic causal models. Neuroimage 22, 1157-1172.

Penny, W. D., Stephan, K. E., Mechelli, A., and Friston, K. J. (2004b). Modelling functional integration: a comparison of structural equation and dynamic causal models. Neuroimage 23(Suppl. 1), S264-S274.

Pereira, J. M., Williams, G. B., AcostaCabronero, J., Pengas, G., Spillantini, M. G., Xuereb, J. H., Hodges, J. R., and Nestor, P. J. (2009). Atrophy patterns in histologic vs clinical groupings of frontotemporal lobar degeneration. Neurology 72, 1653-1660.

Price, C. J., Crinion, J., and Friston, K. J. (2006). Design and analysis of fMRI studies with neurologically impaired patients. J. Magn. Reson. Imaging 23 , 816-826.

Price, C. J., and Friston, K. J. (1999) Scanning patients with tasks they can perform. Hum. Brain Mapp. 8 , 102-108.

Ramnani, N., Behrens, T. E., Penny, W. and Matthews, P. M. (2004). New approaches for exploring anatomical and functional connectivity in the human brain. Biol. Psychiatry 56 613-619.

Riera, J. J., Wan, X., Jimenez, J. C., and Kawashima, R. (2006). Nonlinear local electrovascular coupling. I: A theoretical model. Hum. Brain. Mapp. 27, 896-914.

Rodriguez-Oroz, M. C., Jahanshahi, M. Krack, P., Litvan, I., Macias, R., Bezard, E., and Obeso, J. A. (2009). Initial clinical manifestations of Parkinson's disease: features and pathophysiological mechanisms. Lancet Neurol. $8,1128-1139$.

Roebroeck, A., Formisano, E., and Goebel, R. (2005). Mapping directed influence over the brain using Granger causality and fMRI. Neuroimage 25, 230-242.

Roebroeck, A., Formisano, E., and Goebel, R. (2009a). The identification of interacting networksin the brain usingfMRI: model selection, causality and deconvolution. Neuroimage doi: 10.1016/j. neuroimage.2009.1009.1036.

Roebroeck, A., Formisano, E., and Goebel, R. (2009b). Reply to Friston and David after comments on: the identification of interacting networks in the brain using fMRI: mode selection, causality and deconvolution. Neuroimage doi: 10.1016/j. neuroimage.2009.1010.1077.

Rohrer, J. D., Ridgway, G. R., Modat, M., Ourselin, S., Mead, S., Fox, N. C., Rossor, M. N., and Warren, J. D. (2010). Distinct profiles of brain atrophy in frontotemporal lobar degeneration caused by progranulin and tau mutations. Neuroimage doi: $10.1016 / \mathrm{j}$ neuroimage.2009.12.088.

Rowe, J., Friston, K., Frackowiak, R., and Passingham, R. (2002a). Attention to action: specific modulation of corticocortical interactions in humans. Neuroimage 17, 988-998.

Rowe, J., Stephan, K. E., Friston, K., Frackowiak, R., Lees, A., and Passingham, R. (2002b). Attention to action in Parkinson's disease: impaired effective connectivity among frontal cortical regions. Brain 125, 276-289.

Rowe, J. B., Eckstein, D., Braver, T., and Owen, A. M. (2008a). How does reward expectation influence cognition in the human brain? J. Cogn Neurosci. 20, 1980-1992.

Rowe, J. B., Hughes, L., Ghosh, B. C., Eckstein, D., Williams-Gray, C. H., Fallon, S., Barker, R. A., and Owen, A. M. (2008b). Parkinson's disease and dopaminergic therapy - differential effects on movement, reward and cognition. Brain 131, 2094-2105

Rowe, J. B., Hughes, L., Williams-Gray, C. H., Bishop, S., Fallon, S., Barker, R. A., and Owen, A. M. (2008c). The val(158)met COMT polymorphism's effect on atrophy in healthy aging and Parkinson's disease. Neurobiol. Aging. 31, 1064-1068.

Rowe, J. B., Hughes, L. E., Barker, R. A., and Owen, A. M. (2010). Dynamic causal modelling of effective connectivity from fMRI: are results reproducible and sensitive to Parkinson's disease and its treatment? Neuroimage 31, 1064-1068.

Rowe, J. B., Sakai, K., Lund, T. E., Ramsoy, T., Christensen, M. S., Baare, W. F., Paulson, O. B., and Passingham, R. E. (2007). Is the prefrontal cortex necessary for establishing cognitive sets? J. Neurosci. 27, 13303-13310.

Rowe, J. B., Siebner, H., Filipovic, S. R., Cordivari, C., Gerschlager, W. Rothwell, J., and Frackowiak, R. (2006). Aging is associated with contrasting changes in local and distant cortical connectivity in the human motor system. Neuroimage $32,747-760$.

Sabatini, U., Boulanouar, K., Fabre, N., Martin, F., Carel, C., Colonnese, C. Bozzao, L., Berry, I., Montastruc, J. L., Chollet, F., Rascol, O. (2000). Cortical motor reorganization in akinetic patients with Parkinson's disease: a functional MRI study. Brain 123 394-403.

Sakai, K., and Passingham, R. E. (2003). Prefrontal interactions reflect future task operations. Nat. Neurosci. 6 75-81.

Sakai, K., and Passingham, R. E. (2006). Prefrontal set activity predicts rule- 
specific neural processing during subsequent cognitive performance. $J$. Neurosci. 26, 1211-1218.

Sapir, A., Kaplan, J. B., He, B. J., and Corbetta, M. (2007). Anatomical correlates of directional hypokinesia in patients with hemispatial neglect. J. Neurosci. 27, 4045-4051.

Schuyler, B., Ollinger, J. M., Oakes, T. R., Johnstone, T., and Davidson, R. J. (2009). Dynamic causal modeling applied to fMRI data shows high reliability. Neuroimage 49 , 603-611.

Seeley, W. W., Crawford, R. K., Zhou, J., Miller, B. L., and Greicius, M. D. (2009). Neurodegenerative diseases target large-scale human brain networks. Neuron 62, 42-52.

Sharma, N., Baron, J. C., and Rowe, J. B. (2009). Motor imagery after stroke: relating outcome to motor network connectivity. Ann. Neurol. 66, 604-616.

Smith, J. F., Pillai, A., Chen, K., and Horwitz, B. (2010a). Identification and validation of effective connectivity networks in functional magnetic resonance imaging using switching linear dynamic systems. Neuroimage 52, 1027-1040.

Smith, S., Woolrich, M., and Miller, K. (2010b). "Evaluation of network connectivity methods for resting fMRI timeseries," in Poster presented at the 16th Annual Meeting of the Organization for Human Brain Mapping, Barcelona (poster 1013).
Smith, S. M., Fox, P. T., Miller, K. L., Glahn, D. C., Fox, P. M., Mackay, C. E., Filippini, N., Watkins, K. E., Toro, R., Laird, A. R., Beckman, C. F. (2009). Correspondence of the brain's functional architecture during activation and rest. Proc. Natl. Acad. Sci. U.S.A. 106, 13040-13045.

Sonty, S. P., Mesulam, M. M., Weintraub, S., Johnson, N. A., Parrish, T. B., and Gitelman, D. R. (2007). Altered effective connectivity within the language network in primary progressive aphasia. J. Neurosci. 27, 1334-1345.

Soto, C., and Estrada, L. D. (2008). Protein misfolding and neurodegeneration. Arch. Neurol. 65, 184-189.

Stephan, K. E., Kamper, L., Bozkurt, A., Burns, G. A., Young, M. P., and Kotter, R. (2001). Advanced database methodology for the Collation of Connectivity data on the Macaque brain (CoCoMac). Philos. Trans. R. Soc. Lond. B Biol. Sci. 356, 1159-1186.

Stephan, K. E., Kasper, L., Harrison, L. M., Daunizeau, J., den Ouden, H. E., Breakspear, M., and Friston, K. J. (2008). Nonlinear dynamic causal models for fMRI. Neuroimage 42, 649-662.

Stephan, K.E., Penny, W. D., Daunizeau, J., Moran, R. J., and Friston, K. J. (2009a). Bayesian model selection for group studies. Neuroimage 46, 1004-1017.

Stephan, K. E., Tittgemeyer, M., Knosche, T. R., Moran, R. J., and Friston, K. J. (2009b). Tractography-based priors for dynamic causal models. Neuroimage 47, 1628-1638.
Stephan, K. E., Penny, W. D., Moran, R. J., den Ouden, H. E., Daunizeau, J., and Friston, K. J. (2010). Ten simple rules for dynamic causal modeling. Neuroimage 49, 3099-3109.

Supekar, K., Menon, V., Rubin, D. Musen, M., and Greicius, M. D. (2008). Network analysis of intrinsic functional brain connectivity in Alzheimer's disease. PLoS Comput. Biol. 4, e1000100. doi: 10.1371/journal.pcbi.1000100.

Verdon, V., Schwartz, S., Lovblad, K. O., Hauert, C. A., and Vuilleumier, P. (2010). Neuroanatomy of hemispatial neglect and its functional components: a study using voxel-based lesion-symptom mapping. Brain 133, 880-894.

Vincent, J. L., Patel, G. H., Fox, M. D. Snyder, A. Z., Baker, J. T., Van Essen, D. C., Zempel, J. M., Snyder, L. H., Corbetta, M., and Raichle, M. E. (2007). Intrinsic functional architecture in the anaesthetized monkey brain. Nature 447, 83-86.

Vul, E., Harris, C., Winkielman, P., and Pashler, H. (2009). Puzzlingly high correlations in $\mathrm{AMRI}$ studies of emotion, personality, and social cognition (The paper formerly known as "Voodoo correlations in social neuroscience"). Perspect. Psychol. Sci. 4, 274-290.

Witt, S. T., and Meyerand, M. E. (2009). The effects of computational method, data modeling, and TR on effective connectivity results. Brain Imaging Behav. 3, 220-231.
Wu, T., Wang, L., Chen, Y., Zhao, C., Li, K., and Chan, P. (2009). Changes of functional connectivity of the motor network in the resting state in Parkinson's disease. Neurosci. Lett. 460, 6-10.

Zhou, J., Greicius, M. D., Gennatas, E. D., Growdon, M. E., Jang, J. Y. Rabinovici, G. D., Kramer, J. H., Weiner, M., Miller, B. L., and Seeley, W. W. (2010). Divergent network connectivity changes in behavioural variant frontotemporal dementia and Alzheimer's disease. Brain 133, 1352-1367.

Conflict of Interest Statement: The author declares that the research was conducted in the absence of any commercial or financial relationships that could be construed as a potential conflict of interest.

Received: 16 July 2010; paper pending published: 11 August 2010; accepted: 27 August 2010; published online: 17 September 2010.

Citation: Rowe JB (2010) Connectivity analysis is essential to understand neurological disorders. Front. Syst. Neurosci. 4:144. doi:10.3389/fnsys.2010.00144 Copyright (c) 2010 Rowe. This is an open-access article subject to an exclusive license agreement between the authors and the Frontiers Research Foundation, which permits unrestricted use, distribution, and reproduction in any medium, provided the original authors and source are credited. 